\title{
LOS MACROINVERTEBRADOS ACUÁTICOS Y LA CALIDAD BIOLÓGICA DEL AGUA EN UNA QUEBRADA ANDINA, ANTIOQUIA-COLOMBIA.
}

\author{
Andrés Felipe Escobar Gutiérrez ${ }^{1^{\star}}$, Yimmy Montoya Moreno ${ }^{2}$ \\ 1 Ingeniero Sanitario Universidad de Antioquia, Medellín, Colombia, e-mail: afelipe.escobar@udea.edu.co \\ 2 Doctor en Ingeniería, Investigador grupo GeoLimna, Universidad de Antioquia. e-mail: \\ yimmymontoya3@hotmail.com

\section{RESUMEN}

La quebrada La Chaparrala surte al acueducto municipal de Andes-Antioquia, en 2014 se realizó la caracterización fisicoquímica y biológica de sus aguas. La turbiedad, DQO, alcalinidad, nitratos, N-amoniacal, nitrógeno total, fósforo total, ortofosfatos y dureza total, mostraron variabilidad alta y sobrepasaron los límites de calidad admisibles para el consumo humano. Se encontraron 37 géneros de insectos acuáticos pertenecientes a 27 familias y cinco phyla. Los dípteros fueron el orden más abundante con un $25.9 \%$ del total de individuos y las familias Chironomidae (38.77\%), Baetidae $(21.03 \%)$, Simuliidae $(12.03 \%)$ y Physidae $(7,74 \%)$ representan un $79.56 \%$ de todos los organismos encontrados. Aunque el cuerpo de agua presenta una calidad biológica dudosa (BMWP/Col medio $=46.33$, EPT $=48.83 \%$ ), se evidencia una disminución progresiva en la calidad del agua, se observó la presencia de las familias Leptophlebiidae, Perlidae e Hydropsychidae que habitan en aguas limpias y oxigenadas. Se evidenció que la quebrada aún es capaz de auto depurarse.

Palabras clave: Macroinvertebrados acuáticos; Calidad del agua; Variables fisicoquímicas; Índices ecológicos.

Recibido: 21 de enero de 2019. Aceptado: 16 de Mayo de 2019

Received: January 21, 2019 Accepted: May 16, 2019

\section{AQUATIC MACROINVERTEBRATES AND THE BIOLOGICAL QUALITY OF THE WATER IN LA CHAPARRALA STREAM, ANDES MUNICIPALITY (ANTIOQUIA), COLOMBIA.}

\begin{abstract}
La Chaparrala river is the most representative in Andes (Antioquia), in 2014 the physicochemical and biological characterization of the water was performed. The turbidity, COD, alkalinity, nitrate, $\mathrm{N}$-ammonia, total nitrogen, total phosphorus, orthophosphate and total hardness showed high variability and exceeded the bounds of acceptable quality for human consumption. 37 genera of aquatic insect belonging to 27 families and five phyla were found. Diptera were the most abundant order with $25.9 \%$ of the total and the families Chironomidae (38.77\%), Baetidae (21.03\%), Simuliidae (12.03\%) and Physidae $(7.74 \%)$ account for $79.56 \%$ of all organisms founded. Although the body of water has a dubious biological quality (BMWP $/$ medium $\mathrm{Col}=46.33$, EPT $=$ 48.83\%) and shows a progressive decline in water quality, we observed the presence of families Leptophlebiidae, Perlidae and Hydropsychidae who live in clean and oxygenated water. It was evident that the river is still capable of self-purification.
\end{abstract}

Keywords: Aquatic macroinvertebrates, Water quality, Physicochemical variables, Ecological indexes.

Cómo citar este artículo: A. Escobar, Y. Montoya. "Los macroinvertebrados acuáticos y la calidad biológica del agua en una quebrada Andina, Antioquia-Colombia", Revista Politécnica, vol. 15, no.29 pp.65-81, 2019. DOI: 10.33571/rpolitec.v15n29a6 


\section{INTRODUCCIÓN}

Cada día se agudizan los problemas asociados con la contaminación de los cuerpos de agua, por ende se agrava la situación de los organismos acuáticos que se enfrentan al hombre y a la amenaza de un recurso cada vez más escaso. Paralelamente a la creciente demanda de agua de buena calidad por parte de la población humana y para el uso agrícola, la contaminación orgánica ha conducido a la reducción del recurso debido al deterioro de su calidad [1].

En las últimas décadas los ecosistemas acuáticos continentales son los que más han sufrido los impactos causados por la actividad humana, donde los desechos industriales y domésticos tienen como destino final los ríos, y en último término, el mar [2]. Las relaciones de abundancia y distribución han llevado a que se incremente el interés por el estudio del recurso hídrico [3].

Dentro de los indicadores de estado ambiental que son usados para un sistema acuático se deben considerar además de las variables fisicoquímicas, el uso de las comunidades biológicas, las cuales interactúan de manera dinámica y cambiante. Tanto los parámetros físicos como químicos presentes en el agua, permiten percibir ideas sobre las condiciones del medio durante el momento, pero no para establecer fluctuaciones espacio temporales [4]. En contraste, los organismos bénticos reflejan no solo el estado de las condiciones actuales sino, también los cambios durante amplios periodos debido a la poca movilidad y extensos ciclos de vida; uno de los componentes bióticos más usados para evaluar la calidad ambiental de los reoambientes son los macroinvertebrados, objeto de análisis de este estudio. Ya que los macroinvertebrados dan cuenta del estado ecológico en periodos cortos, dado sus ciclos de vida y los peces dan cuenta de cambios (espacio- temporales) en el estado ecológico en periodos de tiempo más largos [4].

La bioindicación a partir de los macroinvertebrados acuáticos tiene varias ventajas: facilidad en la identificación de los organismos, amplio set de ríos evaluados en el país, estudios realizados desde los años 80 , facilidad para conseguir bibliografía y para el caso de Antioquia y la zona andina, un alto ajuste a los valores de bioindicación, ya que fueron generados para esta región del país.
Un alto porcentaje de los estudios realizados en el país han sido generados en Antioquia. Entre los trabajos recientes elaborados en la zona andina el del Área Metropolitana del Valle de Aburrá [5], lleva varios años con el proyecto RedRío que mediante tres fases proporciona herramientas para el diseño una red de monitoreo ambiental en la cuenca hídrica del Río Aburrá con la implementación del monitoreo de variables bióticas (macroinvertebrados acuáticos), físicas y químicas del agua; adicionalmente, se cuenta con ciertos estudios similares en las subregiones del departamento [6], [7], [8], [9], [10], [11].

En el departamento de Boyacá [12] determinaron la composición y estructura de los macroinvertebrados mediante descriptores como la densidad y biomasa, a fin de evaluar dicha información como indicador local del estado de calidad ambiental de un tramo de quebrada; en el departamento de Cundinamarca, encontraron diferencias en la composición por zona y una reducción progresiva en la riqueza de morfotipos. Además de la variación en la biomasa de los grupos funcionales dominantes relacionada con alteraciones en las condiciones de calidad ambiental del sistema. [13] estudian la estructura y composición de macroinvertebrados acuáticos en un bosque alto andino; en esta misma línea de estudios [14] determinan la calidad del agua del Río Frio mediante el uso de macroinvertebrados acuáticos como bioindicadores al aplicar el método BMWP; en el departamento del Valle del Cauca [15] determinan el impacto del suelo agropecuario sobre macroinvertebrados acuáticos en pequeñas quebradas de la cuenca del río La Vieja, así mismo [16] evalúa la respuesta de los macroinvertebrados bentónicos a las condiciones de la calidad de agua en el tramo medio-alto del Río Felidia, cuenca del Río Cali.

El proyecto denominado calidad física e hidrobiológica del agua en el río San Juan, Andes, Antioquia es la investigación en la que se encuentra enmarcada este estudio y pretende evaluar la calidad fisicoquímica y biológica de la cuenca del río San Juan; con el propósito de aportar a la identificación de los ejes de dinámica ambiental presentes en el área de estudio, es por esta razón que la evaluación de la biota acuática de macroinvertebrados del río San Juan aportará insumos para el esclarecimiento y compresión sistémica del funcionamiento natural y antrópico que 
tiene lugar en este cuerpo de agua. Debido a la creciente presión sobre los ecosistemas acuáticos.

El objetivo de este trabajo es presentar la caracterización de la calidad del agua de la quebrada La Chaparrala, a través del análisis de la comunidad de macroinvertebrados acuáticos, la medición de variables fisicoquímicas y la estimación de índices, que permitan relacionar las condiciones de los recursos hidrobiológicos y las actividades antrópicas en la cuenca.

\section{MATERIALES Y METODOS}

\section{Área de estudio:}

El área de estudio es la quebrada La Chaparrala, se encuentra localizada al Suroeste del departamento de Antioquia, distante de Medellín aproximadamente $121 \mathrm{~km}$ y a una altura entre 2.800 a 1.250 m.s.n.m. (figura 1). Según [11] está quebrada hace parte de la estrella hidrográfica de la Reserva Natural Farallones de Citará y surte al acueducto municipal de Andes.

El cauce de la quebrada tiene un recorrido aproximado de $15.4 \mathrm{~km}$, presenta morfología predominante de escalón - pozo, con la presencia de material en el lecho de rocas, y cantos rodados [17], de acuerdo a la información recogida por el grupo de investigación en gestión y modelación ambiental - GAIA y el grupo de investigación en ingeniería y gestión ambiental - GIGA, la cuenca de la quebrada presenta un área de $37,7 \mathrm{~km}^{2}$, un perímetro de 38.49 $\mathrm{km}$ y un gradiente promedio del cauce $91.2 \mathrm{~m} / \mathrm{km}$ [18]. Entre la parte baja, donde está localizada la mayor parte de la zona cafetera de la cuenca, y la parte alta, cubierta de bosque intervenido se encuentra ganadería de doble propósito con variación en el manejo de praderas, ya que el $21 \%$ se encuentra en pasto enmalezado, mientras el $13 \%$ se encuentra en buen estado como pasto manejado [18].

El régimen de precipitaciones del territorio es bimodal, con periodos de lluvias intensas, en Abril Mayo y Octubre - Noviembre y periodos más secos en Junio - Julio y Enero - Febrero. La precipitación media anual es de $1800 \mathrm{~mm}$, en las partes altas se presentan los valores más elevados, con valores que superan los $2.200 \mathrm{~mm} / a n ̃ o$, porque están bajo la influencia de los vientos provenientes del pacífico, que chocan con los vientos cálidos del cañón del Cauca [17].

\section{Sitios de muestreo:}

Mediante visita previa al área de la microcuenca de la quebrada La Chaparrala, se establecieron tres estaciones de muestreo a 1.436, 1.339 y 1.270 m.s.n.m. Las estaciones de muestreo fueron seleccionadas con base en información levantada en campo, en consideración con los usos del agua y del suelo, cobertura vegetal y las actividades antrópicas que se realizan alrededor de la microcuenca.

Estación 1 (E 1) se estableció en el sitio conocido como La Bocatoma, ubicada en la finca El Molino. Esta estación se encuentra bajo la influencia de cierto grado de contaminación orgánica, donde se percibe el olor a aguas residuales y se da el avistamiento de residuos sólidos, sobre todo en las épocas de cosecha. La estación presenta un sustrato arenoso-pedregoso.

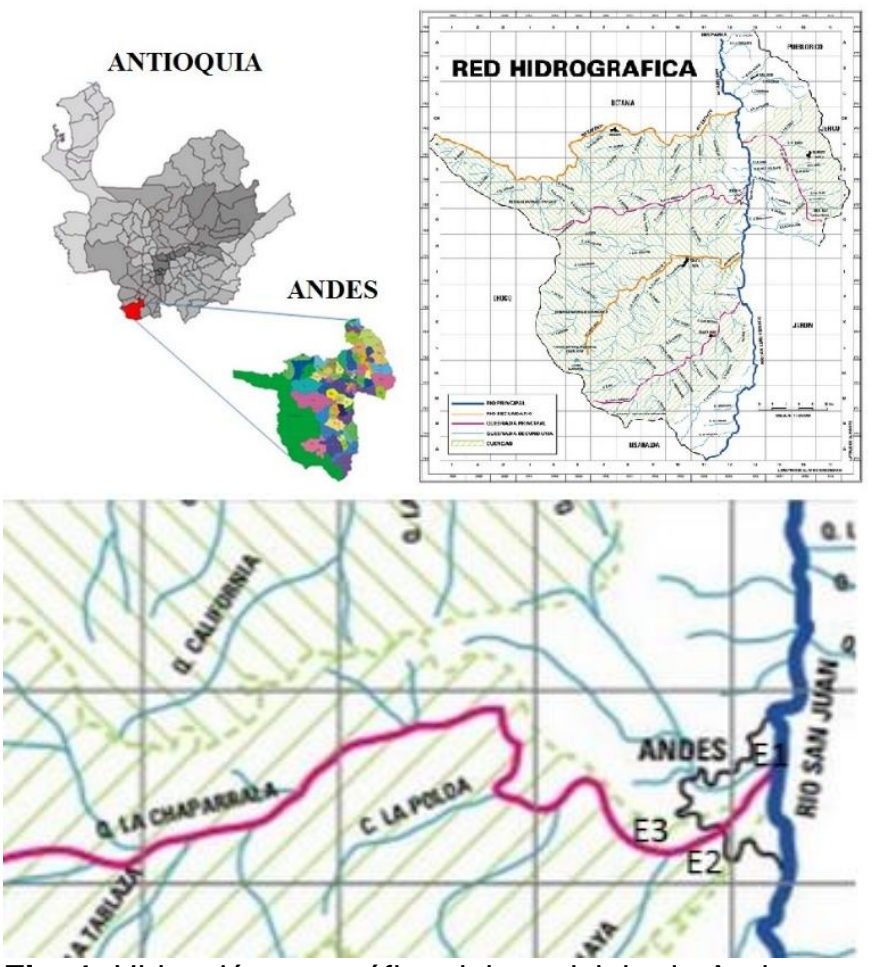

Fig. 1. Ubicación geográfica del municipio de Andes (Antioquia), Colombia en la microcuenca de la quebrada La Chaparrala [fuente: mapa de la red hidrográfica de Andes]

Estación 2 (E 2) Un par de kilómetros más delante de la E1 aguas abajo, se ubicó la segunda estación en el sector aledaño a la cárcel. Se caracteriza por tener una amplia cobertura vegetal donde hay arbustos medianos, pastizales y el lecho de la 
corriente está altamente expuesto a la luz solar; las condiciones organolépticas confirman la colecta de las aguas combinadas del barrio María Auxiliadora, el sector aledaño a la penitenciaría Las Mercedes y las aguas servidas de la planta de potabilización, para verterlas directamente sobre la quebrada. Se tiene la presencia de residuos sólidos en el lecho de la corriente y animales de carroña cercanos al lugar, así mismo, se observa que parte de la población que habita en cercanías a la zona realiza labores domésticas del lavado de ropa. La estación presenta un sustrato heterogéneo arenoso-pedregoso.

Estación 3 (E 3) Aguas abajo de la segunda estación de muestreo, se localizó la tercera estación caracterizada por la presencia de una cobertura vegetal alta, con condiciones organolépticas que permiten conocer que este punto recibe la descarga de las aguas residuales de la planta de sacrificio y de la población aledaña. El sustrato predominante en este sitio es arenoso-pedregoso.

\section{Muestreo:}

Para la recolección de las muestras biológicas y la medición de las variables físicas y químicas del agua, cada estación fue visitada en cuatro ocasiones con una periodicidad trimestral en los meses de febrero, mayo, agosto y octubre de 2014, en diferentes momentos pluviométricos (figura 2).

\section{Medición de variables físicas y químicas}

Simultáneamente a la recolección de macroinvertebrados, en cada estación se tomaron y midieron in situ por duplicado las variables fisicoquímicas y ambientales: temperatura del ambiente, temperatura del agua, oxígeno disuelto, porcentaje de saturación de oxígeno, $\mathrm{CO}_{2}, \mathrm{pH}$, conductividad eléctrica, alcalinidad total, dureza total, sólidos disueltos, demanda química de oxigeno-DQO y turbiedad. La temperatura del agua, oxígeno disuelto, conductividad eléctrica y sólidos disueltos se determinaron in situ con un equipo multiparamétrico. Se tomaron muestras que fueron analizadas en el laboratorio de Ciencias de la Tierra de la seccional del Suroeste de la Universidad de Antioquia por vía espectrofotométrica con la utilización de kits de spectroquant de acuerdo con [19].

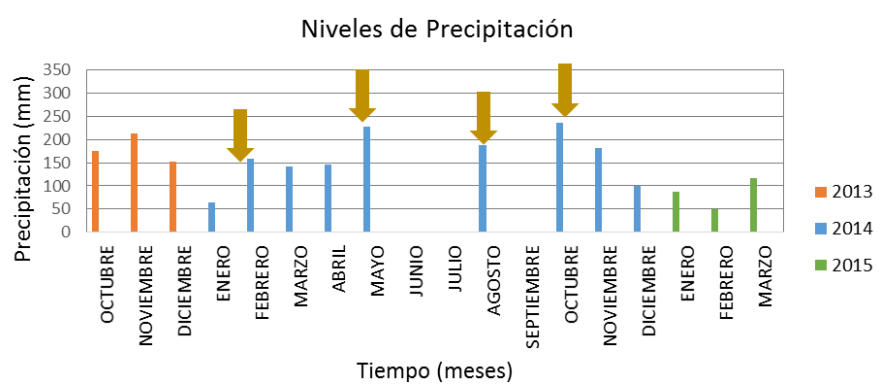

Fig. 2. Régimen pluvial y las campañas de muestreos (flechas). FUENTE: Instituto de Hidrología, Meteorología y Estudios Ambientales (IDEAM).

\section{Colecta de individuos}

La recolección de especímenes acuáticos de las tres estaciones de muestreo (E1, E2, E3) se realizó mediante la implementación de los métodos de recolección propuestos por [5]. Para la colecta de los macroinvertebrados acuáticos, se realizó un muestreo por duplicado en diferentes transectos de la corriente y se empleó una red tipo Surber de $0.22 \mathrm{~m}^{2}$ de área de muestreo, con la cual se obtuvieron las muestras cuantitativas en contracorriente. Se recolectaron los organismos por un periodo de cinco (5) minutos, la totalidad de individuos se denominó como una muestra. Adicionalmente, en la toma de muestras cualitativas se emplearon redes triangulares y de pantalla. También se obtienen muestras cualitativas de las capturas manuales con pinzas de los organismos adheridos a sustratos como piedras, troncos, hojarasca y plantas acuáticas, con un periodo de captura de cinco (5) minutos. Por otro lado, se determinó la velocidad de la corriente, el perímetro húmedo y el caudal del ambiente lotico en cada estación de muestreo.

Los macroinvertebrados acuáticos colectados fueron depositados en viales plásticos y se fijaron en alcohol al $70 \%$, se transportaron al Laboratorio debidamente rotulados con la fecha, coordenadas, el tipo de muestra. Posteriormente fueron separados para su identificación taxonómica, mediante la utilización del estereomicroscopio Karl Zeiss y guías taxonómicas de [20], [21], [22], [23] y [24].

\section{Análisis de los datos}

Los datos obtenidos en los tres muestreos fueron sometidos a un análisis exploratorio mediante el uso 
de estadísticos de tendencia central (media aritmética) y de dispersión (coeficiente de variación relativa de Pearson, C.V). La asociación entre los macroinvertebrados acuáticos y las variables físicas, químicas y microbiológicas se efectuó mediante un análisis de regresión lineal múltiple. Se realizó un análisis de correspondencia canónica rectificado (DCCA) para identificar la longitud del gradiente y luego se hizo un análisis de correspondencia canónica (CCA) en donde se integraron los géneros más importantes, las variables evaluadas y los sitios de colecta, para identificar los patrones de distribución de los macroinvertebrados acuáticos. Los análisis estadísticos mencionados se realizaron con el paquete estadístico Statgraphics Centurion XV y Canoco 5.0.

\section{Índices}

En los análisis de las comunidades de macroinvertebrados se aplicaron índices ecológicos, donde se tienen en cuenta tres elementos fundamentales de la estructura de las comunidades bióticas: riqueza, equidad y abundancia. Entre estos se encuentran el índice BMWP-Col [5], el Índice \% ETP [25] y para el análisis fisicoquímico se empleó el índice de calidad del agua-ICA [26] y [27].

\section{RESULTADOS}

\section{Características Climáticas y Fisicoquímicas}

Se realizaron cuatro muestreos durante el ciclo hidrológico, en el periodo seco que oscila entre los meses de diciembre-febrero, en el periodo de transición de época seca a húmeda entre los meses de marzo-mayo, seguidamente un periodo seco entre junio-julio y finalmente un periodo de cambio de temporada seca a húmeda para los meses de agosto-noviembre de 2014.

Respecto al coeficiente de variación de las variables fisicoquímicas, estas se pueden agrupar en tres grupos: las poco variables (CV <20\%) tales como la temperatura del agua, la conductividad eléctrica, el $\mathrm{pH}$ y la concentración de nitritos. Las de variación intermedia $(20 \%>\mathrm{CV}<50 \%)$ como los sólidos disueltos totales, el oxígeno disuelto, \% saturación de oxígeno, el potencial redox, DQO, alcalinidad, nitrógeno amoniacal, nitrógeno total y dureza. $Y$ las variables de variación alta (CV $>50 \%$ ), tales como turbiedad, nitratos, fósforo total y ortofosfatos (Tabla 1).
Tabla 1. Medidas de tendencia central y de dispersión de las variables físicas.

\begin{tabular}{|c|c|c|c|c|}
\hline Variables Químicas & $\bar{X}$ & Máximo & Mínimo & $\begin{array}{l}\text { C.V } \\
\text { (\%) }\end{array}$ \\
\hline $\begin{array}{l}\text { Temperatura del agua } \\
\left({ }^{\circ} \mathrm{C}\right)\end{array}$ & 19,7 & 25,2 & 17 & 11 \\
\hline $\begin{array}{l}\text { Conductividad } \\
\text { eléctrica }(\mu \mathrm{S} / \mathrm{cm})\end{array}$ & 46,2 & 54 & 28,2 & 18 \\
\hline $\begin{array}{l}\text { Solidos disueltos } \\
\text { totales (mg/L) }\end{array}$ & 43,3 & 54,5 & 28 & 22 \\
\hline Turbiedad (NTU) & 34,6 & 157 & 8,5 & 125 \\
\hline $\begin{array}{l}\text { Oxígeno disuelto } \\
\text { (mg/L) }\end{array}$ & 5,2 & 6,76 & 3,5 & 24 \\
\hline $\begin{array}{l}\text { Saturación de } \\
\text { oxígeno disuelto (\%) }\end{array}$ & 66,2 & 91,2 & 46,2 & 25 \\
\hline $\mathrm{pH}$ (unidades de $\mathrm{pH}$ ) & 7,1 & 7,67 & 6 & 8 \\
\hline Redox (mV) & 228,3 & 324 & 163 & 20 \\
\hline $\mathrm{DQO}\left(\mathrm{mg} \mathrm{O}_{2} / \mathrm{L}\right)$ & 50 & 53 & 2 & 37 \\
\hline $\begin{array}{l}\text { Alcalinidad (mmol } \\
\left.\mathrm{CaCO}_{3} / \mathrm{L}\right)\end{array}$ & 44,6 & 70 & 20 & 33 \\
\hline $\begin{array}{l}\text { Nitrito } \quad \text { (mg } \\
\left.\mathrm{NO}_{2}^{-} / \mathrm{L}\right)\end{array}$ & 0,3 & 0,3 & 0,3 & 0 \\
\hline $\begin{array}{l}\text { Nitratos } \quad(\mathrm{mg} \\
\left.\mathrm{NO}_{3}{ }^{-} / \mathrm{L}\right)\end{array}$ & 1,9 & 5,5 & 0,2 & 98 \\
\hline $\begin{array}{l}\mathrm{N} \text { - amoniacal (mg } \\
\left.\mathrm{NH}_{4}^{+} / \mathrm{L}\right)\end{array}$ & 0,1 & 0,22 & 0,05 & 43 \\
\hline $\begin{array}{l}\text { Nitrógeno total (mg } \\
\mathrm{N} / \mathrm{L} \text { ) }\end{array}$ & 0,8 & 1,55 & 0,5 & 44 \\
\hline Fósforo total (mg P/L) & 0,2 & 0,59 & 0,05 & 94 \\
\hline $\begin{array}{l}\text { Ortofosfato (mg } \\
\left.\mathrm{PO}_{4}{ }^{3-} / \mathrm{L}\right)\end{array}$ & 0,5 & 1,5 & 0,2 & 82 \\
\hline $\begin{array}{l}\text { Dureza total } \\
\left(\mathrm{mgCaCO}_{3} / \mathrm{L}\right)\end{array}$ & 33,2 & 54 & 18 & 40 \\
\hline
\end{tabular}

\section{Variables Biológicas}

En la tabla 2 se presentan los macroinvertebrados recolectados mediante el método cuantitativo durante las campañas de toma de muestras realizadas en los meses de febrero, mayo, agosto y octubre de 2014. En la quebrada La Chaparrala se capturaron un totoal 37 taxas pertenecientes a 27 
familias y 1189 ejemplares de macroinvertebrados acuáticos, pertenecientes a los phylum Platyhelminthes, Nematomorpha, Annelida, Arthropoda y Mollusca.

Del total de taxas el mayor número corresponde a los dípteros con 7 géneros (25.9\%). A este le siguen los ephemeroptera, odonata y trichoptera con 5 géneros $(18.5 \%)$ y coleópteros con 4 géneros $(14.8 \%)$. Las familias que prsentaron mayor abundancia fueron Chironomidae (38.7\%), Baetidae (38.77\%), Simuliidae (21\%) y Physidae (12\%), los cuales representan una fraccion importante de los organismos capturados (79.5\%). Estas familias se presentaron en todas las estaciones de muestreo durante el periodo hidrologico.

Para el mes de Agosto del año 2014 se registró el mayor número de macroinvertebrados acuáticos recolectados durante las campañas de toma de muestras, en las tres estaciones analizadas para la quebrada La Chaparrala, resultados que superan ampliamente el número de individuos registrados en el resto de meses que se llevaron a cabo los análisis.

En la (E1) predominan los dípteros Chironomidae (Chironomidae) y Simulium (Simuliidae), los efemerópteros Lepthohyphes (Tricorythidae), Camelobaetidus y Baetodes (Baetidae), los cuales se relacionan con ambientes de buena calidad con contaminación moderada. En la (E2) sobresalen los dípteros Chironomidae (Chironomidae) y Simulium (Simuliidae), los efemerópteros Baetodes (Baetidae) y Thraulodes (Leptophelebiidae). Con base en las características de los organismos encontrados se puede estimar que en la (E2) se presenta una condicion similar con respecto a la (E1), con una tendencia al incremento del grado de contaminación. En la (E3) predominan los dípteros Chironomidae (Chironomidae), los basommatophora Physa (Physidae) y los efeemeropteros Lepthohyphes (Tricorythidae), Camelobaetidus y Baetodes (Baetidae), los cuales se relacionan con ambientes con presencia de agua con un grado de contaminación moderada a muy contaminada.
En cuanto a la distribución espacial y temporal los taxa Physa, Chironomidae, Simulium, Baetodes, Camelobaetidus, Baetidae, Thraulodes, Lepthohyphes, Corydalus, Smicridea, Girardia estuvieron presentes en todas las estaciones de estudio de la corriente de la quebrada La Chaparrala.

Es importante tambien destacar la presencia exclusiva por estaciones de muestreo; en la estación (E1) cercana a la bocatoma de los taxa Acari, Phyllogomphoides, Progophus y Leptonema, en la estación dos (E2) aledaña al sector de la carcel los taxa Heterelmis, Limonicola, Clogmia, Muscidae y Oecetis, y finalmente los taxa que se presentaron exclusivamente para la estacion tres (E3) en inmediaciones de la planta de sacrificio son Bellardina, Naididae, Hetaerina y Chimarra.

La estación 1 presentó en general los mayores valores de índice de dominancia respecto a las otras dos estaciones de muestreo, con valores de 0,23, $0,25,0,55$ y 0,34 para cada uno de los muestreos. Estos valores son indicadores de ambientes sometidos a tensores ambientales, ya que la zona de la bocatoma presenta una influencia alta del cultivo de café. 
Tabla 2. Macroinvertebrados acuáticos recolectados cuantitativamente durante las campañas de toma de muestras.

\begin{tabular}{|c|c|c|c|c|c|c|c|c|c|c|c|c|c|c|c|c|c|c|c|c|c|c|c|c|c|c|}
\hline \multirow{3}{*}{ Orden } & \multirow{3}{*}{ Familia } & \multirow{3}{*}{ Genero } & \multicolumn{6}{|c|}{ Febrero de 2014} & \multicolumn{6}{|c|}{ Mayo de 2014} & \multicolumn{7}{|c|}{ Agosto de 2014} & \multicolumn{5}{|c|}{ Octubre de 2014} \\
\hline & & & \multicolumn{2}{|c|}{ E1 } & \multicolumn{2}{|c|}{ E2 } & \multicolumn{2}{|c|}{ E3 } & \multicolumn{2}{|c|}{ E1 } & \multicolumn{2}{|c|}{ E2 } & \multicolumn{3}{|c|}{ E3 } & \multirow{2}{*}{$\begin{array}{c}\mathrm{E} 1 \\
\mathrm{M} 2\end{array}$} & \multicolumn{3}{|c|}{ E2 } & E3 & & E1 & & E2 & & E3 \\
\hline & & & M1 & $\mathrm{M} 2$ & M1 & M2 & M1 & M2 & M1 & M2 & M1 & $\mathrm{M} 2$ & M1 & M2 & M1 & & M1 & M2 & M1 & $\mathrm{M} 2$ & M1 & M2 & M1 & $\mathrm{M} 2$ & M1 & M2 \\
\hline Acari & & & & & & & & & & & & & & & & 2 & & & & & & & & & & \\
\hline Racommatonhor & Anylidae & Ferrissia & & & & & & & & & & & & & 1 & & & & & & & & & & 1 & \\
\hline Dasomintatopiord & Physidae & Physa & & 1 & 3 & & & & & & & & & & 10 & 7 & & 6 & 34 & 28 & & & 1 & & 2 & \\
\hline & & Cylloepus & & 1 & & & & & & & & & 1 & & 1 & 1 & & & & & 1 & & & & & \\
\hline & & Disersus & & & & & & & & & & & & & 1 & 2 & & 3 & & & & & & & & \\
\hline Coleoptera & Elmiaae & Heterelmis & & & & & & & & & 1 & & & & & & & & & & & & & & & \\
\hline & & Macrelmis & & 1 & 1 & & & & & & 1 & & & & 1 & & & & & & & & & & & \\
\hline & Blepharoceridae & Limonicola & & & & & & & & & 1 & & & & & & & & & & & & 1 & & & \\
\hline & Chironomidae & Chironomidae sp & 2 & 3 & 2 & 3 & & 1 & & & 1 & & 50 & & 171 & 40 & 2 & 97 & 14 & 47 & 8 & & 14 & & 6 & \\
\hline & Clognia & Clognia sp & & & 2 & & & & & & & & & & & & 1 & & & & & & & & & \\
\hline Diptera & Muscidae & Muscidae sp & & & & 1 & & & & & & & & & & & & & & & & & & & & \\
\hline & Psychodidae & Maruina & & & & & & & & & & & & & 1 & 1 & & & & 1 & & & & & & \\
\hline & Simuliidae & Simulium & & 2 & 2 & 1 & & 1 & & 9 & 8 & & & & 21 & 3 & 35 & 21 & 2 & 2 & 6 & & 22 & & 8 & \\
\hline & Tipulidae & Bellardina & & & & & 1 & & & & & & & & & & & & & & & & & & & \\
\hline & & Baetodes & 7 & & & & & & 7 & 9 & 30 & & 8 & & 3 & 3 & & 7 & 4 & 5 & 5 & & 18 & & 7 & \\
\hline & Baetidae & Camelobaetidus & & & 1 & & 1 & & & 3 & 1 & & 27 & & 2 & 2 & 2 & & 3 & 28 & 28 & & 13 & & 8 & \\
\hline Ephemeroptera & & & 1 & & & & & & 1 & 1 & 3 & & 5 & & & & & & 3 & 4 & & & & & & \\
\hline & Leptophelebiidae & Thraulodes & 1 & 1 & 5 & 7 & 9 & 10 & & 1 & & & & & 1 & 1 & 13 & 2 & & & & & & & & \\
\hline & Tricorythidae & Lepthohyphes & 12 & 25 & 1 & & 5 & 4 & & 1 & 1 & & & & & & & & & & & & & & & \\
\hline Gordiodidae & Chordodidae & Chordodidae sp & & & & & & & & & & & & & & 2 & & & 2 & & & & & & & \\
\hline & Haplotaxidae & Haplotaxidae $s p$ & & & & & & & & & & & & & & & & & & & 1 & & & & & \\
\hline Haplotaxida & Naididae & Naididae sp & & & & & & & & & & & 1 & & & & & & & & & & & & & \\
\hline & & & & & 1 & & 1 & & & & & & & & & & & & & & & & & & & \\
\hline Mesogastropoda & Hydrobiidae & Hydrobiidae $s p$ & & & & & & & & & 1 & & & & & & & & & & & & & & & \\
\hline Neuroptera & Corydalidae & Corydalus & & 1 & & 1 & & 1 & & 1 & & & & & & & & & & & & & & & & \\
\hline & Calopterigyda & Hetaerina & & & & & 1 & 2 & & & & & & & & & & & & & & & & & & \\
\hline & Gomphidae & Phyllogomphoides & 1 & 1 & & & & & & & & & & & & & & & & & & & & & & \\
\hline Odonata & Gomphidae & Progophus & 2 & 1 & & & & & & & & & & & & & & & & & & & & & & \\
\hline & Libellulidae & Erythrodiplax & & 2 & & & & 1 & & & & & & & & & & & & & & & & & & \\
\hline & Libenuniaje & Macrothemis & & & & & & & & 1 & & & & & & & & & 1 & & 2 & & & & 4 & \\
\hline Plecoptera & Perlidae & Anacroneuria & 3 & 2 & & & 1 & & & 1 & & & & & & & & & & & & & & & & \\
\hline & Helicopsychidae & Helicopsyche & & & & & & & & & & & & & 1 & 1 & & & 1 & & & & & & & \\
\hline & Hydronsychidae & Leptonema & & & & & & & & 1 & & & & & 1 & 1 & & & & & & & & & & \\
\hline Trichoptera & Hydropsychidae & Smicridea & 14 & 16 & 1 & 2 & & & & & & & & & & & & & & & & & & & 2 & \\
\hline & Leptoceridae & Oecetis & & & & 2 & & & & & & & & & & & & & & & & & & & & \\
\hline & Philopotamidae & Chimarra & & & & & & & & & & & & & & & & & & & & & & & 1 & \\
\hline Tricladida & Planariidae & Girardia & & & & & & & & & 4 & & & & 5 & & 2 & & 3 & 10 & & & 2 & & 4 & \\
\hline & SUBTOTAL & & 1 & 00 & 3 & & 39 & & 36 & & & 2 & & 92 & & 286 & & 191 & & 192 & & 51 & & 71 & & 43 \\
\hline & TOTAL INDIVIDUOS & & & & 1 & & & & & & & 180 & & & & & & 669 & & & & & & 65 & & \\
\hline
\end{tabular}




\section{Índices Ecológicos.}

Los valores obtenidos para los índices ICA, $\mathrm{BMWP} / \mathrm{Col}$ y \% ETP. Se evidencia que los valores del ICA sobreestiman la calidad del agua en casi todos los escenarios, mientras que el \% ETP evidencia un panorama subestimado (Tabla 3).

Tabla 1. Valores de los índices BMWP/Col, ICA y el \% ETP para cada estación y mes de muestreo.

\begin{tabular}{|c|c|c|c|c|}
\hline Mes & Estación & ICA & BMWP/Col & \%ETP \\
\hline \multirow{6}{*}{$\begin{array}{c}\text { Febrero } \\
\text { (Periodo } \\
\text { seco) }\end{array}$} & & 0.78 & 70 & $82 \%$ \\
\hline & E1 & (Buena) & (Aceptable) & $\begin{array}{l}\text { (Muy } \\
\text { buena) }\end{array}$ \\
\hline & E2 & 0.76 & 43 & $53 \%$ \\
\hline & L2 & (Buena) & (Dudosa) & (Buena) \\
\hline & & 0.76 & 44 & $77 \%$ \\
\hline & E3 & (Buena) & (Dudosa) & $\begin{array}{l}\text { (Muy } \\
\text { buena) }\end{array}$ \\
\hline \multirow{5}{*}{$\begin{array}{c}\text { Mayo } \\
\text { (Periodo } \\
\text { lluvioso) }\end{array}$} & & 0.89 & 56 & $69 \%$ \\
\hline & E1 & (Buena) & (Dudosa) & (Buena) \\
\hline & F? & 0.88 & 45 & $67 \%$ \\
\hline & LL & (Buena) & (Dudosa) & (Buena) \\
\hline & E3 & --- & $\begin{array}{c}17 \\
\text { (Critica) }\end{array}$ & $\begin{array}{c}43 \% \\
\text { (Regular) }\end{array}$ \\
\hline \multirow{6}{*}{$\begin{array}{l}\text { Agosto } \\
\text { (Periodo } \\
\text { lluvioso } \\
\text { leve) }\end{array}$} & & 0.82 & 70 & $6 \%$ \\
\hline & E1 & (Buena) & (Aceptable) & (Mala) \\
\hline & $E_{2}$ & 0.82 & 45 & $13 \%$ \\
\hline & 22 & (Buena) & (Dudosa) & (Mala) \\
\hline & E3 & 0.82 & 41 & $25 \%$ \\
\hline & & (Buena) & (Dudosa) & (Regular) \\
\hline \multirow{6}{*}{$\begin{array}{l}\text { Octubre } \\
\text { (Periodo } \\
\text { lluvioso) }\end{array}$} & & 0.77 & 33 & $65 \%$ \\
\hline & E1 & (Buena) & (Critica) & (Buena) \\
\hline & E2 & 0.74 & 37 & $44 \%$ \\
\hline & & (Aceptable) & (Dudosa) & (Regular) \\
\hline & & 0.79 & 55 & $42 \%$ \\
\hline & E3 & (Buena) & (Dudosa) & (Regular) \\
\hline \multirow{2}{*}{\multicolumn{2}{|c|}{ Promedio }} & 0.80 & 46.33 & $48.83 \%$ \\
\hline & & (Buena) & (Dudosa) & (Regular) \\
\hline
\end{tabular}

La DQO mostró correlaciones positivas con el $\mathrm{N}$-amoniacal $(r=0,72, p=0,01)$, el fósforo total $(r=0,82, p=0,004)$ y los ortofosfatos $(r=0,89$, $p=0,011)$, y negativa respecto a los nitritos $(r$ $=-0,82, p=0,0088)$. El índice ICA exhibió una correlación positiva con los nitratos $(r=0,75$, $\mathrm{p}=0,0235)$ y una correlación negativa con los ortofosfatos $(r=-0,9, p=0,017)$; por otra parte, para el índice ETP se presentaron correlaciones negativas con las turbiedad $(r=-$ $0,75, p=0,012)$ y el potencial redox $(r=-0,72$, $p=0,017)$. El índice BMWP no presentó ninguna correlación significativa con las variables evaluadas.

Las variables que no presentaron variación espacial fueron la temperatura del agua, temperatura del aire, conductividad, turbiedad, alcalinidad, $\mathrm{N}$-amoniacal, nitrógeno total, fósforo total, ortofosfatos y los índices de diversidad, dominancia, equidad, similitud y $\mathrm{BMWP} / \mathrm{Col}$. Las variables que presentaron variación temporal fueron: oxígeno disuelto $(F$ $=25.57, p=0,0001)$, saturación de oxígeno $(F$ $=39, p=0), p H(F=3,4, p=0,0012)$, potencial redox $(F=18,1, p=0,0012), D Q O(F=36,5, p$ $=0)$, nitratos $(F=25.57, p=0,0001)$, y los índices ICA $(F=22,4, p=0,0008)$ y ETP $(F=$ 53.8, $\mathrm{p}=0,011)$.

De las variables analizadas, la única que presentó tanto variación espacial $(F=41,8, p=$ 0,0481 ) como temporal $(F=92,5, p=0,023)$ fue la dureza total. El hecho de que no se presentó variación espacial en la mayoría de variables estudiadas, da cuenta de la poca heterogeneidad del ambiente.

El CCA generó un porcentaje de varianza total explicado del $33 \%(p=0.02)$, por lo tanto se sugiere que la correlación entre los macroinvertebrados y las variables ambientales fue significativa. Para este análisis se usaron 9 variables de las cuales resultaron significativas la demanda bioquímica de oxígeno, la turbidez y la dureza. En el biplot del CCA (figura 3) se observa que el primer eje canónico (0.797) mostró en su área positiva una fuerte relación entre el $\mathrm{pH}$, el amonio y la DQO; por su parte, en el área negativa se registró una importante relación entre la turbidez y la conductividad con la mayoría de taxa, estaciones y muestreos. No hay una segregación entre las condiciones ambientales de las estaciones, sólo un aislamiento en el periodo seco. 
Los taxa Thraulodes, Smicridea, Hetaerina, Erythrodiplax, Anacroneuria, Clogmia y Oecetis se asocian al periodo seco con la DQO, Namoniacal y $\mathrm{pH}$. Estos taxa se aíslan solo en el periodo seco evidenciando aguas oligotróficas a mesotróficas, las cuales dan razón de presencia de vegetación sumergida, fondo lodoso, en ocasiones se encuentran contaminadas y con materia orgánica en descomposición en el cuerpo de agua.

En relación con el índice de Shannon-Weaver (Tabla 4) se presentan valores bajos respecto al índice de diversidad, con resultados menores a la unidad durante todos los muestreos y en todas las estaciones. La E1 durante el mes de agosto presenta el valor más bajo, mientras que para el mes de febrero la E2 presenta el máximo valor encontrado para este índice comunitario.

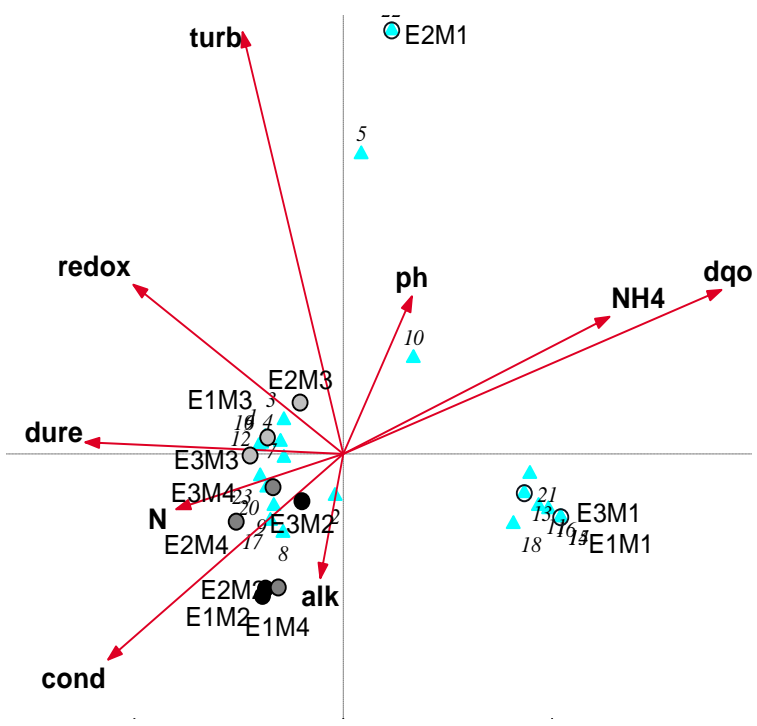

Fig. 3. CCA triplot entre la comunidad de macroinvertebrados, variables ambientales y estaciones y momentos de muestreo. Los puntos representan las tres estaciones y cuatro muestreos, las líneas rojas simbolizan las variables ambientales y los íconos azules los taxa de macroinvertebrados, los cuales son los siguientes: 1. Physa, 2. Cylloepus, 3. Disersus, 4. Chironomidae sp, 5. Clognia sp, 6. Maruina, 7. Simulium, 8. Baetodes, 9. Camelobaetidus, 10. Thraulodes, 11. Lepthohyphes, 12. Chordodidae sp, 13. Hetaerina, 14. Phyllogomphoides, 15. Progophus, 16. Erythrodiplax, 17. Macrothemis, 18. Anacroneuria, 19. Helicopsyche, 20. Leptonema, 21. Smicridea, 22. Oecetis, 23. Girardia. E1, E2 y E3: estaciones 1, 2 y 3. M1, M2, M3 y M4: muestreos 1, 2, 3 y 4 respectivamente. Cond: conductividad eléctrica, dure: dureza total, alk: alcalinidad, turb: turbiedad y $\mathrm{N}$ : nitratos.

Con respecto al índice de Dominancia de Simpson es importante señalar, que los valores obtenidos en las diferentes campañas de muestreo manifiestan una tendencia cercana entre los datos, sin embargo, los valores más alejados se encuentran en la estación tres (E3) con un valor mínimo de 0.117 para el mes de Octubre de 2014 y en la estación uno (E1) con un valor máximo de 0.555 en el mes de Agosto de 2014. Para el índice de equidad de Pielou la distribución indica una tendencia uniforme por medio de valores cercanos a uno, lo que indica que todas las especies son igualmente abundantes, como se muestra en la Tabla 4.

Tabla 2. Índices de Diversidad, Dominancia y Equidad.

\begin{tabular}{|c|c|c|c|c|}
\hline \multicolumn{2}{|c|}{$\begin{array}{c}\text { ESTACIÓN } \\
\text { Y FECHA } \\
\text { DE } \\
\text { MUESTREO }\end{array}$} & $\begin{array}{c}\text { Diversidad } \\
\text { Shannon- } \\
\text { Weaver }\end{array}$ & $\begin{array}{c}\text { Dominancia } \\
\text { Simpson }\end{array}$ & $\begin{array}{c}\text { Equidad } \\
\text { de } \\
\text { Pielou }\end{array}$ \\
\hline & E1 & 0.809 & 0.232 & 0.688 \\
\hline \multirow[t]{3}{*}{$\begin{array}{l}\text { Febrero } \\
\text { de } 2014\end{array}$} & E2 & 0.947 & 0.138 & 0.850 \\
\hline & E3 & 0.711 & 0.283 & 0.683 \\
\hline & E1 & 0.726 & 0.254 & 0.726 \\
\hline \multirow[t]{3}{*}{$\begin{array}{c}\text { Mayo } \\
\text { de } 2014\end{array}$} & E2 & 0.651 & 0.356 & 0.625 \\
\hline & E3 & 0.504 & 0.386 & 0.647 \\
\hline & E1 & 0.496 & 0.555 & 0.412 \\
\hline \multirow[t]{3}{*}{$\begin{array}{l}\text { Agosto } \\
\text { de } 2014\end{array}$} & E2 & 0.573 & 0.360 & 0.600 \\
\hline & E3 & 0.730 & 0.236 & 0.701 \\
\hline & E1 & 0.600 & 0.339 & 0.709 \\
\hline \multirow[t]{2}{*}{$\begin{array}{l}\text { Octubre } \\
\text { de } 2014\end{array}$} & E2 & 0.679 & 0.223 & 0.803 \\
\hline & E3 & 0.911 & 0.117 & 0.911 \\
\hline
\end{tabular}




\section{DISCUSIÓN}

El régimen pluviográfico es una consecuencia del desplazamiento en sentido norte-sur, de la zona de convergencia intertropical a lo largo del año, la cual es característica de la zona tropical. Frecuentemente se presentan en la zona, lluvias de tipo convectivo, principalmente en las horas de la tarde, los cuales son intensas, pero de corta duración [11]. La precipitación anual en la región presenta variaciones importantes, desde los $1600 \mathrm{~mm}$ en el municipio de Valparaiso, hasta los 3200 $\mathrm{mm}$ en los Farallones del Citará.

En el año se presentan en promedio entre 180 y 240 días de lluvias, resaltando los meses de diciembre y enero, como los más secos, y los meses de mayo y octubre los más lluviosos en la región [28].

Los valores encontrados para la conductividad se encuentran entre el rango de valores normales para este parámetro, los cuales están entre 30 y $60 \mu \mathrm{S} / \mathrm{cm}$, resultados por debajo de estos valores indican baja productividad, y valores por encima indican alta productividad. Varios autores [10], [15] y [29] afirman que el aumento de esta variable está estrechamente ligada con la afectación negativa de la calidad del agua por acciones antrópicas, ya que la E1 pese a ser la parte alta, exhibe una presión alta debida al cultivo del café.

Los sólidos disueltos totales evidencian un claro aumento de este parámetro hacia la desembocadura de La quebrada la Chaparrala en el río San Juan con un coeficiente de variación (C.V.) del $22 \%$ que es indicador de la estabilidad del sistema, por otra parte, dichos aumentos en los valores obtenidos permiten establecer que estos incrementos son debidos, a las aguas residuales descargadas $y$ actividades antrópicas de la zona que afectan la vida acuática, en donde se aumenta la productividad y se disminuye la diversidad de especies [16], [29] y [30].

La turbiedad se relaciona con la presencia de materia orgánica, inorgánica, arcilla, lodo y plancton [10]; presentó un C.V. alto del $125 \%$, posiblemente atribuido a las lluvias registradas en la zona durante los días de muestreo y previos a los mismos. Pese a esto, autores como [30], [31], mencionan que el aumento de la turbiedad en el agua tiene efectos nocivos directos sobre la fauna acuática en donde se limita el paso de la luz a través del agua y se afecta la productividad de las aguas.

El C.V. del $24 \%$ para la concentración de oxígeno disuelto indica que el sistema puede presentar variaciones en el entorno, el cual puede sufrir disminuciones de las concentraciones de oxígeno disuelto debido a que el consumo de oxígeno se incrementa paralelamente con la temperatura y contenido de materia orgánica [10],[32]; las variaciones que se presentan durante el mes de Agosto pueden atribuirse a estos factores, además este hallazgo coincide con la época de recolección y lavado de café mencionado para la parte alta de una quebrada de condiciones similares [33].

El pH en la quebrada La Chaparrala presenta un C.V bajo del $8 \%$, valores que se encontraron dentro del límite aceptable para la vida acuática y que generalmente se aceptan en un rango óptimo de $\mathrm{pH}$ para las especies de agua dulce entre 6.5 y 9.0 unidades [31].

El potencial redox en los puntos de muestreo caracteriza la presencia de reacciones de oxidación principalmente de materia orgánica de la quebrada, lo que indica que los organismos heterótrofos utilizan el $\mathrm{O}_{2}$ como receptor de electrones [33], es posible apreciar una tendencia en la que el potencial redox de la Bocatoma aguas abajo se incrementa, al llegar la estación aledaña a la cárcel y aguas abajo de esta misma se presenta una disminución en la desembocadura al río San Juan, incidencia igualmente descrita por [31].

La presencia de nitratos se relaciona típicamente con los lixiviados desde los campos cultivados y se mueve a poca profundidad, subterráneamente, hacia las fuentes superficiales; esta lixiviación se reduce hasta en un $15 \%$ cuando se dan prácticas de manejo de conservación de suelos y agua [30]. El aporte de nitratos se favorece por el uso de abonos nitrogenados [38].

Los ortofosfatos presentan un comportamiento similar al obtenido con las mediciones del fósforo total presente en las corrientes con fluctuaciones y aumentos en la concentración de este parámetro principalmente en la estación dos (E2). En los ecosistemas no alterados los ortofosfatos varían normalmente entre 0.033 y $0.099 \mathrm{mg} / \mathrm{l}$ [10]. En la E2 predominaron Chironomidae, Simullium $y$ Baetodes, los cuales son considerados como parcialmente tolerantes a la contaminación [34], [38]. 
En la quebrada La Chaparrala los macroinvertebrados más abundantes son de la familia Chironomidae que presentan un amplio rango de saprobiedad, no obstante el género oligosaprobio Simulium y los que se caracterizan por un rango ambiental de oligosaprobio a ligeramente mesosaprobio como los efemerópteros Baetodes, Camelobaetidus y Thraulodes y el tricóptero Smicridea se encuentran en abundancia. Aunque en esta corriente, en el trayecto de la estación de la planta de sacrificio se identificó un organismo en abundancia que prefiere ambientes mesosapobios (Physa), puede ser considerado como organismo influyente para evidenciar un deterioro de la calidad de agua en esta corriente e indicador de la afectacion del recurso [38] reportan la misma situación en numerosos ambientes lóticos colombianos. La composicion de los macroinvertebrados frente al grado de saprobiedad del medio indica que en los sitios de muestreo de la quebrada La Chaparrala se presentan buenas condiciones ambientales, pese a que en esta corriente hídrica se le realizan aportes significativos de contaminanción que afectan la calidad del recurso.

Los resultados más bajos del BMWP se podrían explicar a través de la presencia masiva de Chironomidae sp, Physa y Girardia y en parte a la granulometria del fondo, dado que en las épocas de muestreo el material granular fino fue el sustrato predominate sobre la corriente de la quebrada La Chaparrala [7]. Es de suponer la influencia de este valor sobre los resultados de diversidad estimados. Los puntajes del BMWP/Col obtenidos a partir de las familias de macroinvertebrados acuáticos, de acuerdo a los criterios de [34] indican aguas ligeramente contaminadas a moderadamente contaminadas para las cuales la calidad del recurso hídrico oscila entre aceptable y dudosa, este índice sólo toma en cuenta algunas taxa, ya que aún no se tiene valores de bioindicación para todos los grupos, de ahí la discrepancia entre estos valores y demás valores obtenidos con los demás índices desarrollados. Los valores de los índices de bioindicación obtenidos, evidencian que las aguas en cuestión presentan una buena calidad pero demuestran un aumento en la proliferación de contaminación hacia estas corrientes, con menor calidad en el muestreo de mayo-periodo lluvioso y mejor calidad biológica del agua en agosto-periodo lluvioso leve.

El índice \% ETP sólo toma en cuenta organismos pertenecientes a los órdenes
Ephemeroptera, Trichoptera y Plecoptera, los cuales son muy sensibles a la contaminación, pues requieren aguas con muy buena oxigenación [34], dan cuenta que los valores más altos significan aguas más oxigenadas y limpias, por lo que es posible observar que el valor promedio de este índice refleja una calidad regular de la corriente de la quebrada La Chaparrala con cierto grado de afectación durante la mayor parte de los estudios realizados, este índice presenta durante el mes de agosto los valores más críticos registrados, debido a que los organismos involucrados son menos tolerantes entre otras cosas, a la contaminación con materia orgánica.

El índice ICA en general presentó resultados de buena calidad para el análisis de los tramos que componen el cuerpo de agua de la quebrada La Chaparrala como objeto de estudio, caracterizando como apto el recurso hídrico, lo que permite disponer de este para el consumo humano. No obstante se puede decir que estos resultados presentan limitaciones en la descripción de las características del río, puesto que los parámetros que se evalúan son muy reducidos y puede haber pérdida de información sobre contaminación que puede estar dada por otros factores que no se toman en cuenta dentro del índice, de igual forma una de las limitaciones del índice radica en que los recursos hídricos diferentes pueden caer en una misma categoría de calidad numérica, aunque el tipo de contaminación y su origen sean significativamente diferentes [27]. Además, una de las variables consideradas cuyo factor de peso es igual al de los demás factores, es el oxígeno disuelto; un parámetro que puede ser muy poco afectado si se ejerce algún tipo perturbación, ya que las condiciones naturales del río contribuyen a que los niveles de oxígeno sean siempre elevados. Un aspecto relevante es que no evalúa la presencia de coliformes, por lo que este resultado es parcial.

Al realizar la comparación entre todos los índices, el ICA presentó la menor sensibilidad, ya que son muestras puntuales que pueden no reflejar las condiciones que registran mejor los organismos acuáticos, por lo que si descartamos este índice; la relación entre el índice BMWP/Col y \% ETP mantiene cierta afinidad y una tendencia a determinar la calidad de la corriente de manera muy similar, pero \% ETP es más estricto al definir la calidad de las estaciones por sólo tener en cuenta la presencia de organismos poco tolerantes a la contaminación del medio acuático y que requieren de aguas oxigenadas, poco mineralizadas, con $\mathrm{pH}$ circunneutral y de baja 
concentración de nutrientes. Hay que tener en cuenta que índices como BMWP/Col y \% ETP se basan en la tolerancia de las taxas a ciertos parámetros fisicoquímicos y condiciones autóctonas; estas preferencias ecológicas de las especies pueden variar según las condiciones de la región, por lo que los valores de calidad pueden variar si se hace una calibración de estos índices a condiciones propias de la zona en estudio.

Se encontró con base en los análisis estadísticos que un grupo de los taxa de macroinvertebrados se asocian con las variables químicas como el potencial redox (Disersus sp), nitratos (Leptonema sp, Girardia $s p)$, conductividad eléctrica (Camelobaetidus $s p$, Macrothemis $s p$ ), dureza (Simulium $s p$, Chordodidae $s p$ ) y $\mathrm{pH}$ (Thraulodes $s p$ ) para las diferentes estaciones de muestreo. Esta asociación de macroinvertebrados y variables químicas es posible por las condiciones físicas y por los principales factores que gobiernan la abundancia y distribución de macroinvertebrados acuáticos [33], [35], [38] en la zona de estudio.

Entre los géneros que sobresalen, Thraulodes ha sido asociado a ambientes con un sustrato constituido principalmente por grava en menor proporción, por arena y hojarasca [36]. De igual manera está relacionado con aguas de buena calidad a ligeramente contaminadas [10]. El incremento en su abundancia se ha asociado a zonas de bosque [39], ya que es altamente sensible a disminuciones de oxígeno y a contaminantes [40].

Los organismos del género Smicridea usualmente viven en aguas limpias, oxigenadas, debajo de piedras, troncos y material vegetal acumulado, habitan en sustrato dominado por roca, grava, arena y hojarasca, vegetación arbórea en las riberas de la corriente [9], aunque estos son organismos que habitan en ambientes de buena calidad generalmente, también fue posible relacionarlos en zonas que recibe vertimientos directos de aguas residuales domésticas [36], lechos de baja pendiente y cobertura natural ribereña moderada, zonas donde se cultiva plátano y se desarrollan actividades de ganadería en presencia de materia orgánica fina utilizada como fuente de alimentación [37].

Para los géneros Clogmia sp que habita en depósitos de agua en las brácteas de muchas plantas y en orificios de troncos viejos [21], aguas estancadas [38] y Erythrodiplax sp que se encuentra en corrientes poco profundas, rodeados de abundante vegetación acuática sumergida o emergente [21] y Oecetis sp que residen en lechos de pendiente moderada, con cobertura de vegetación natural ribereña y aledaño a cultivos de café [37], la naturaleza física del sustrato es uno de los factores que determina la abundancia y distribución de estas especies en la biota acuática. Es de resaltar, que en este estudio se encontró este género bajo condiciones de fluctuación alta del caudal.

Las condiciones de la quebrada La Chaparrala durante la primera campaña de muestreo presentaron una calidad ambiental del agua de oligosaprobia a mesosaprobia, donde se registró un leve índice de contaminación, que se corrobora con los parámetros químicos asociados a estos muestreos y la constante indicación de eutrofización de la quebrada en su trayecto hacia el río San Juan por la continua presencia de cobertura vegetal y descargas de aguas residuales. La DQO, la conductividad eléctrica y la turbiedad exhiben los vectores de mayor impacto en el sistema, lo que puede ser indicador del tipo de contaminación que impacta al ecosistema y que reduce la calidad biológica del agua.

Finalmente, el índice BMWP/Col no presentó correlaciones significativas con ninguna variable. Tampoco en el análisis multivariado, por lo que se considera que aporta mucha información, ya que no hay solapamiento de información. Se plantea que la Quebrada Chaparrala es un sistema torrencial con crecientes súbitas, lo que limita el desarrollo de la comunidad de macroinvertebrados acuáticos, reduciendo su capacidad bioindicadora de la calidad biológica del agua, no obstante, los índices ICA y EPT parecen sobreestimar la calidad del agua.

\section{CONCLUSIONES}

Las concentraciones de la mayoría de las variables fisicoquímicas como la temperatura del agua, conductividad eléctrica, SDT, oxígeno disuelto, \% de saturación de oxígeno, $\mathrm{pH}$, potencial redox, DQO, alcalinidad, dureza total, algunas especies de nitrógeno y fosforo total se encontraron dentro de los rangos permisibles para aguas de consumo humano, excepto variables como la turbiedad, nitritos y ortofosfatos, las cuales reflejaron una disminución gradual en la calidad del agua por contaminación antrópica, especialmente por actividades del sector cafetero y agrícola.

Variables fisicoquímicas como la turbiedad, DQO, alcalinidad, nitratos, $\mathrm{N}$-amoniacal, nitrógeno total, fósforo total, ortofosfatos y 
dureza total evidencian continuas fluctuaciones y aumentos en los niveles admisibles de calidad para abastecimiento humano, propicios para el deterioro de la calidad de la corriente a lo largo del gradiente longitudinal, sin embargo, a pesar de estos niveles y fluctuaciones de contaminación por actividades antrópicas, la corriente hídrica aún es capaz de autopurificarse y generar un efecto buffer de amortiguación.

La distribución espacio temporal de macroinvertebrados acuáticos en la quebrada la Chaparrala a lo largo de su perfil longitudinal presenta una abundancia destacada de géneros como Simullium, Baetodes, Camelobaetidus, Thraulodes y Smicridea que frecuentan un rango ambiental amplio de oligosaprobio a ligeramente mesosaprobio, no obstante se pudo identificar en el tramo final de la corriente un organismo abundante, característico de ambientes mesosapobios (Physa), organismo que manifiesta un deterioro del recurso y afectacion de la calidad.

Respecto a los índices comunitarios, es posible determinar una similaridad media entre las estaciones de toma de muestra, lo cual se puede evidenciar en el bajo valor en la dominancia y la alta diversidad de especies.

En relación con los valores encontrados para el índice biótico BMWP/Col es posible determinar que la calidad hidrobiológica de la quebrada La Chaparrala en las diferentes campañas de muestreo presenta un rango de calidad aceptable a crítica a medida que se desciende desde la parte alta de la quebrada hasta su desembocadura, sin embargo, es posible apreciar la capacidad amortiguadora y de recuperación intrínseca de la corriente para fluctuar de una calidad critica a aceptable, hecho tangible que se registró durante el último periodo de muestreo y análisis.

El índice ICA modificado tiene en cuenta una amplia gama de factores químicos en el ambiente que caracterizan la calidad del cuerpo de agua, la corriente superficial evidencia un rango de jerarquía de calidad buena a aceptable, en consecuencia es apta para disponer del recurso para el abastecimiento humano. El índice BMWP/Col se presenta como el mejor indicador de la calidad biológica del agua, por lo que se recomienda su uso para la evaluación y diagnóstico del estado ecológico en este cuerpo de agua. No obstante, idealmente se debería realizar esta evaluación mediante el uso de diatomeas, para acotar los resultados obtenidos.
La hipótesis de que la quebrada La Chaparrala presenta un gradiente de contaminación se acepta, ya que a lo largo del gradiente longitudinal se tiene que las estaciones presentan mayor deterioro subsecuentemente, es decir que el gradiente de contaminación es proporcional, por lo que se puede afirmar que la fuente de agua presenta un continuo estrés generado por la contaminación independientemente de que este cuerpo de agua exhiba la capacidad de reponerse en ciertos trayectos. Aunque, el gradiente es inverso, ya que debido a la presencia de cultivos de café se encontró menor calidad del agua en la parte alta, la cual se recupera en la parte media.

En general, la quebrada La Chaparrala como fuente de abastecimiento principal del acueducto urbano del municipio de Andes tiene una buena calidad fisicoquímica e hidrobiológica, a pesar de que los resultados obtenidos muestran una tendencia notable de contaminación, por lo que demanda la implementación de medidas preventivas para el mejoramiento y calidad del recurso.

\section{AGRADECIMIENTOS}

Los autores agradecen el apoyo de la Universidad de Antioquia, quien mediante la convocatoria de regionalización permitió realizar esta investigación.

\section{REFERENCIAS BIBLIOGRÁFICAS}

[1] Caicedo, O. Los macroinvertebrados benticos y la contaminación orgánica. Actual Biol.,69, 61-73. 1989.

[2] Álvarez, S.M. y L. Pérez. Evaluación de la calidad de agua mediante la utilización de macroinvertebrados acuáticos en la subcuenca del Yeguare, Honduras: Tesis, Escuela Agrícola Panamericana, Zamorano. 69 p.2007

[3] Betancur Vargas, T. Atlas hidrogeológico del bajo cauca Antioqueño. Medellín. 103p. 2014.

[4] Bojsen, B.H. y Barriga, R. Effects of deforestation on fish community structure in Ecuadorian Amazon streams. Freshwater Biol. 47:2246-2260. 2002.

[5] Red Río. Red de monitoreo ambiental en la cuenca hidrográfica del Río Aburrá - 
Medellín en jurisdicción del área metropolitana FASE IV. Medellín. Área Metropolitana del Valle de Aburrá, 2014.

[6] Posada-García, J.A., Abril-Ramírez, G. y Parra-Sánchez, L.N. Diversidad de los macroinvertebrados acuáticos del páramo de Frontino (Antioquia, Colombia). Caldasia 30 (2): 441-445. 2008.

[7] Garcia, J. y Carmona, J. Caracterización de la calidad del agua de la parte alta de la microcuenca de La Quebrada La cimarronas, EL Carmen De Viboral (Antioquia), utilizando macroinvertebrados acuáticos [Trabajo de pregrado], Medellín. Universidad de Antioquia. 2011.

[8] Montoya Moreno, Y. y Acosta, Y. Análisis de la calidad del Río negro y sus principales tributarios empleando como indicadores los índices ICA , EL BMWP / COL y EL ASPT. Caldasia, 33(1) 193-210. 2011.

[9] Montoya Moreno, Y., Aguirre, N. J., Caicedo, O. y Palacio, J. Dinámica multianual de los macroinvertebrados acuáticos bentónicos en la Quebrada Vegas De La Clara. Investigación, Biodiversidad y Desarrollo, 29(2) 201210. 2010.

[10]Caicedo, O y Palacio, J. Los macroinvertebrados bénticos y la contaminación orgánica en la Quebrada La Mosca (Guarne, Antioquia, Colombia). Actualidades Biológicas, 20, 61-73. 1998.

[11] Corantioquia. Análisis integral del territorio. En Plan de gestión ambiental regional. 81p. 2007.

[12] Motta, Á., Ortega, L., Niño, Y., Pulido, K. y Alcibiades, E. Variación espacial en la estructura y grupos funcionales de los macroinvertebrados acuáticos en la quebrada La Chapa, Santana, Boyacá. Ciencia En Desarrollo, 3, 49-68. 2011.

[13] Ospina Bautista, F., Estévez Varón, J., y Betancur, J. Estructura y composición de la comunidad de macroinvertebrados acuáticos asociados a Tillandsia turneri BAKER (Bromeliaceae) en un bosque alto andino colombiano. Acta Zoológica Mexicana, 20(1), 153-166. 2004.
[14] Bastidas Gálvez, J., Beltrán, J., Cárdenas, A., y Miranda, C. Determinación de la calidad del agua del Río Frío (Cundinamarca) mediante el uso de macroinvertebrados acuáticos como bioindicadores, aplicando el método BMWP. UNI CIENCIA, 1-20. 2014.

[15] Giraldo, L. P., Chará, J., Zúñiga, M., Chará, A. M. y Pedraza, G. Impacto del uso del suelo agropecuario sobre macroinvertebrados acuáticos en pequeñas quebradas de la cuenca del río La Vieja. Revista de Biología Tropical, 203-219. 2014.

[16] Llano Castillo, C. Respuesta de los macroinvertebrados bentónicos a las condiciones de calidad de agua en el tramo medio-alto del Río Felidia, cuenca deL RIOO CALI, Valle del CaucaColombia. Universidad Autónoma de Occidente. 2012.

[17] Espitia, R. C., David, J., García, L., y García, P. Geometría Escalón - Pozo En Ríos De Alta Pendiente. XXII Congreso latinoamericano de hidráulica. Guyana, Venezuela. 2006.

[18] GAIA, G., y GIGA, G. Caracterización cualitativa y cuantitativa de la calidad y cantidad del recurso hídrico superficial en la Cuenca del río San Juan. Medellín. 2002.

[19] APHA, AWWA, WEF. Standard Methods for examination of water and wastewater. 22nd ed. Washington: American Public Health Association; 1360 pp. ISBN 978087553-013-0. 2012.

[20] McCaffertty, W. P. Aquatic Entomology. Boston: Science Books International. 1981.

[21] Roldán Pérez, G. Guía para el estudio de los macroinvertebrados acuáticos del departamento de Antioquia. Medellin. 1988.

[22] Merritt, R. y K. Cummins. An Introduction to the Aquatic Insects of North America. Dubuque: Kendall/Hunt Publishers. 1996.

[23] Fernández, H. R. \& E. Domínguez (Eds.) Guía para la determinación de los artrópodos bentónicos sudamericanos. 
Universidad Nacional de Tucumán. 282 pp. 2001.

[24] Posada, J. A. y Roldán, G. Clave ilustrada y diversidad de las larvas de Trichoptera en el nor-occidente de Colombia. Caldasia 25 (1): 169-192. 2003.

[25] Klemm, D.J., P.A. Lewis., F. Fulk y J.M. Lazorchak. Macroinvertebrate field and laboratory methods for evaluating the biological integrity of surface waters. EPA/600/4-90/030.US.Enviromental

Protection. Cincinnati, Ohio 45268. 253p. 1990.

[26] IDEAM. Calidad del agua superficial en Colombia. In Estudio nacional del agua 2010 pp. 230-280. 2010.

[27] IDEAM. Instituto de Hidrología, Meteorología y Estudios Ambientales. Hoja metodológica del indicador Índice de calidad del agua (Versión 1,00). Sistema de Indicadores Ambientales de Colombia - Indicadores de Calidad del agua superficial. 2011.

[28] E.S.P, I. T. S. P. S. Plan de Saneamiento y Manejo de Vertimientos Municipio de Andes - Antioquia. 2012.

[29] Nubia, V., Caicedo, O., y Aguirre, N. J. Análisis de calidad de agua de la quebrada La Ayurá con base en variables fisicoquímicas y macroinvertebrados acuáticos. Producción + Limpia, 4, 44-60. 2009.

[30] Mejia, M. R. Análisis de la calidad del agua para consumo humano y percepción local de las tecnologías apropiadas para su desinfección a escala domiciliaria, en la microcuenca El Limón, San Jerónimo, Honduras. Centro Agronomico Tropical de Investigación y Enseñanza. 2005.

[31] Montoya, M., Agudelo, R., Ramirez, J., Zapata, P., y Restrepo, F. Quebrada La Chaparrala: calidad del agua desde algunas variables fisicoquímicas. 2014.

[32] Aguirre, J. F. Validación de los indicadores biológicos (macroinvertebrados) para el monitoreo de la cuenca del río Yanuncay. Universidad Politécnica Salesiana. 2011.
[33] Montoya Moreno, Y. Caracterización de la biodiversidad acuática y de la calidad de las aguas de la Quebrada Los Andes, El Carmen de Viboral, Antioquia. Revista Institucional Universidad Tecnológica Del Chocó: Investigación, Biodiversidad Y Desarrollo, 27(1), 85-91. 2008.

[34]Roldán Pérez, G. Los macroinvertebrados y su valor como indicadores de la calidad del agua. Revista Academica Colombiana de Ciencias Exactas, Fisicas Y Naturales, 23, 375-387. 1999.

[35] Rincón, M. E. Comunidad de insectos acuáticos de la quebrada Mamarramos (Boyacá, Colombia). Revista Colombiana de Entomología, 28, 1001-108. 2002.

[36] Quintero, O. C., Jaime, N., y Ramírez, A. Quebrada La Popala: una análisis de calidad del agua desde algunas variables fisicoquímicas, microbiológicas y los macroinvertebrados acuáticos. Gestión $Y$ Ambiente, (1), 85-94. 2011.

[37] Pérez Vera, C. A., \& Muñoz Quesada, F. Composición y estructura de la comunidad de trichoptera (insecta) en un gradiente altitudinal, en el municipio de Andes (Antioquia), Colombia. Universidad de Antioquia. 2011.

[38] Roldán, G., \& Ramírez JJ. Fundamentos de Limnología Neotropical. Editorial Universidad de Antioquia, Medellín.2008.

[39] Lorion, C. M., y Kennedy, B.P. 2009. Riparian forest buffers mitigate the effects of deforestation on fish assemblages in tropical headwater streams. Ecological Applications, 19 (2), 468-479.

[40] Zuñiga, M. C., Rojas, A. M., y Mosquera, S. 1997. Biological aspects of Ephemeroptera in rivers of Southwestern Colombia (South America). In P. Landolt \& M. Sartori (Eds.), Ephemeroptera and Plecoptera: Biology, Ecology, Systematics (pp. 261-268). Fribourg, Switzerland: MTL Mauron + Tinguely \& Lachat SA. 
